\title{
VISIR-VLT high-resolution study of the extended emission of four obscured post-AGB candidates *
}

\author{
M. W. Blanco Cárdenas ${ }^{1}$, M. A. Guerrero ${ }^{1}$, G. Ramos-Larios ${ }^{2}$, L. F. Miranda ${ }^{3,4}$, E. Lagadec $^{5}$, \\ O. Suárez ${ }^{6}$, and J. F. Gómez ${ }^{1}$
}

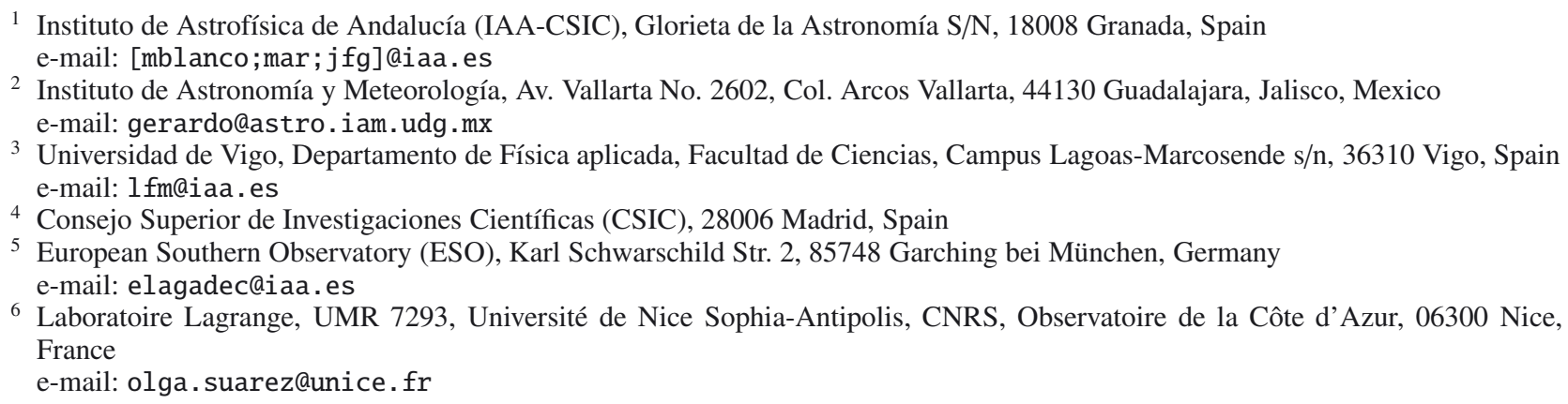

Received 19 September 2012 / Accepted 6 December 2012

\section{ABSTRACT}

\begin{abstract}
Context. The onset of the asymmetry of planetary nebulae (PNe) is expected to occur during the late asymptotic giant branch (AGB) and early post-AGB phases of low- and intermediate-mass stars. Among all post-AGB objects, the most heavily obscured ones might have escaped the selection criteria of previous studies that detected extreme axysimmetric structures in young PNe.

Aims. Since the most heavily obscured post-AGB sources can be expected to descend from the most massive PN progenitors, these should exhibit clear asymmetric morphologies. High-resolution observations of these sources should reveal marked bipolar morphologies, confirming the link between progenitor mass and nebular morphology.

Methods. We have obtained VISIR-VLT mid-IR images of a sample of four heavily obscured post-AGB objects that are barely resolved in previous Spitzer IRAC observations in order to analyze their morphology and physical conditions across the mid-IR. The images obtained in four different mid-IR filters have been deconvolved, flux-calibrated, and used to construct RGB composite pictures as well as color (temperature) and optical depth maps that allow us to study the morphology and physical properties of the extended emission of the sources in our sample.

Results. We have detected the extended emission from the four objects in our sample and resolved it into several structural components that are greatly enhanced in the temperature and optical depth maps. The morphologies of the sample, as well as their physical conditions, reveal the presence of asymmetry in three young PNe (IRAS 15534-5422, IRAS 17009-4154, and IRAS 18454+0001), where the asymmetries can be associated with dusty torii and slightly bipolar outflows. The fourth source (IRAS 18229-1127), a possible post-AGB star, is better described as a rhomboidal detached shell.

Conclusions. The heavily obscured sources in our sample do not show extreme axisymmetric morphologies. This is at odds with the expectation of highly asymmetrical morphologies in post-AGB sources descending from massive PN progenitors, which is otherwise supported by observations of bright mid-IR unobscured sources. The sources presented in this paper may be sampling critical early phases in the evolution of massive PN progenitors, before extreme asymmetries develop.
\end{abstract}

Key words. instrumentation: high angular resolution - methods: observational - stars: AGB and post-AGB - circumstellar matter planetary nebulae: general

\section{Introduction}

Evolved low- and intermediate-mass stars $\left(0.8 M_{\odot}<M<8 M_{\odot}\right)$ undergo heavy mass-loss episodes during the red giant and asymptotic giant branch (AGB) phases. At the tip of the AGB, these stars will eject most of their stellar envelopes in a short time until they evolve into post-AGB stars, the immediate precursors of planetary nebulae (PNe). PNe display an impressive variety of morphologies, from spherical to the most complex shapes: bipolars and multipolars with point-symmetric structures and collimated jets moving at high velocities. It has been

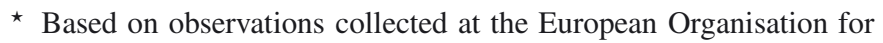
Astronomical Research in the Southern Hemisphere, Chile. Program: 087.D-0367(A). suggested that asymmetric PNe are the rule, rather than the exception (Manchado et al. 2000; Schwarz et al. 1993). The shaping mechanism of complex PNe is a matter of intense debate and has not been completely understood (Balick \& Frank 2002)

It has been claimed that the short transition between the AGB and post-AGB phase contains the clues to understanding the transformation of the spherical stellar envelope into an asymmetric PN (Sahai \& Trauger 1998). AGB stars are surrounded by thick and compact circumstellar envelopes that are rich in dust, and this makes their detection almost impossible at optical wavelengths, whereas their infrared emission is strong. As they evolve into the post-AGB phase, the temperature of the stellar cores increases and the envelopes become optically thin, although it must be noted that it can be found sources at this stage 


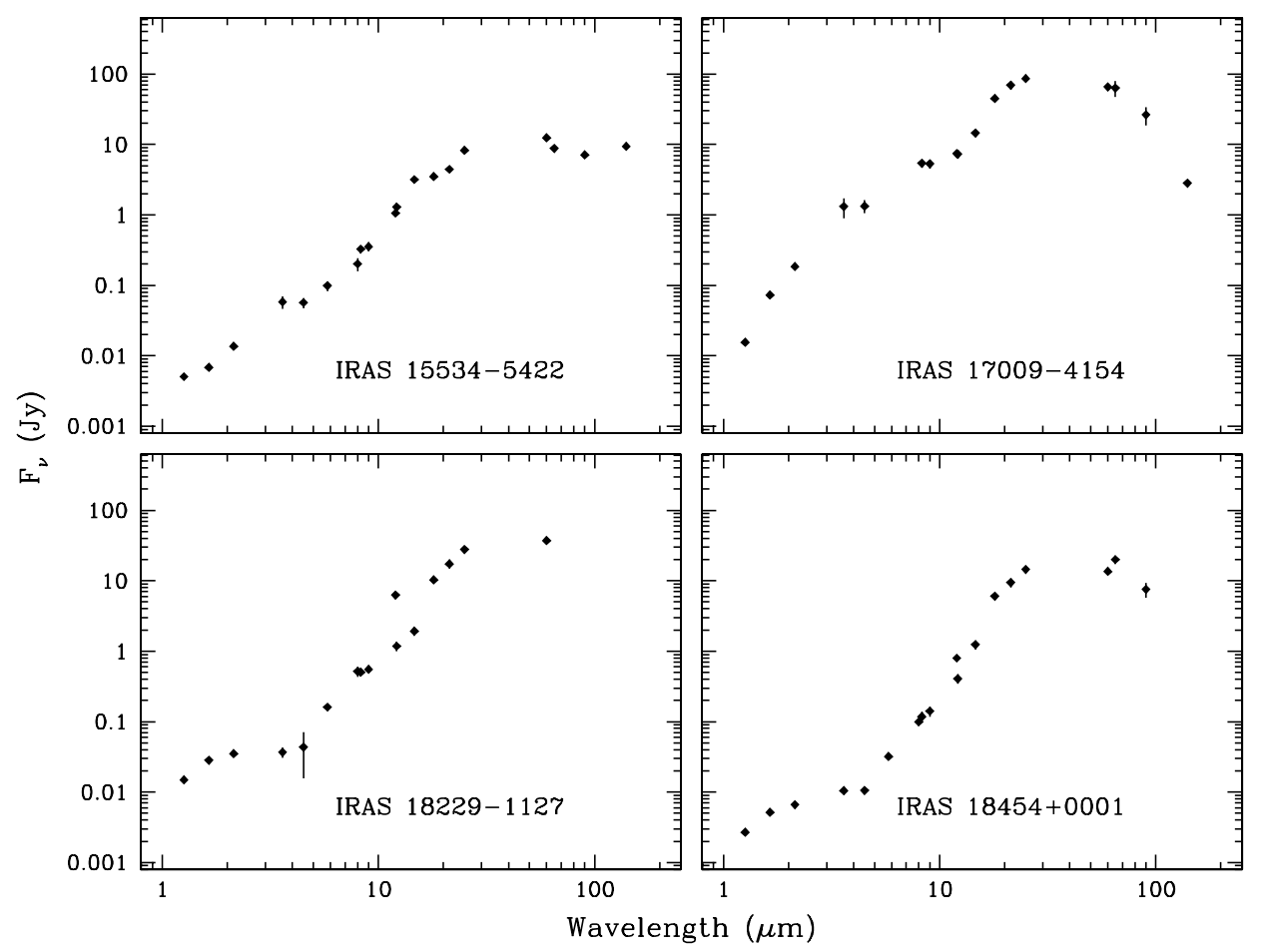

Fig. 1. Near- and mid-IR spectral energy distributions (SEDs) of the sources in our sample. See Ramos-Larios et al. (2009); Ramos-Larios et al. (2012) for further details on the different datasets used to build these SEDs.

and even young PNe that are significantly obscured because they preserve optically thick envelopes (e.g., IRAS 17347-3139, de Gregorio-Monsalvo et al. 2004). Observational studies of the reflection, thermal dust, and ionized emission around proto-PNe and young PNe (Sahai \& Trauger 1998; Sahai et al. 2007; Ueta et al. 2000; Lagadec et al. 2011; Sahai et al. 2011) typically detect the existence of extreme axisymmetric morphologies, with highly collimated bipolar lobes and equatorial rings or torii. On the other hand, spherical and elliptical morphologies seem to be rare among the objects in this transition phase.

Infrared surveys (e.g., 2MASS, IRAS, and AKARI) have contributed to the study of objects in the late AGB and early post-AGB stages, providing the first insights into this short transition phase (e.g., García-Lario et al. 1997; Jiménez-Esteban et al. 2006; Cox et al. 2011). Recently, Ramos-Larios et al. (2009); Ramos-Larios et al. (2012, hereafter RL09 and RL12, respectively) have investigated a sample of heavily obscured postAGB and PNe candidates selected according to their IRAS colors and the lack of an optical counterpart (Suárez et al. 2006) using near-IR $J H K$ and Spitzer IRAC GLIMPSE images, and MSX, AKARI, and IRAS photometric data. RL09 and RL12 have gathered a small sample of four objects that are resolved in Spitzer IRAC GLIMPSE images, namely IRAS 15534-5422, IRAS 17009-4154, IRAS 18229-1127, and IRAS 18454+0001. The spatial resolution of these images $\left(\sim 2^{\prime \prime}\right)$, however, is not adequate to investigate the angularly small structures typical of sources in these evolutionary phases (Suárez et al. 2011; Lagadec et al. 2011). Mid-IR observations using the new generation of ground-based telescopes provide a unique opportunity to resolve the extended emission detected in these four highly obscured post-AGB objects in their transition to the PN phase.

In this paper we present mid-IR VISIR-VLT high angular resolution observations of these four sources. The images have been used to describe their morphologies, to analyze their innermost structures, and to derive color (temperature) and optical depth maps. We next describe the sample in Sect. 2, the observations and data reduction in Sect. 3, and present the results in Sect. 4. The results are discussed in Sect. 5 and a short conclusion is provided in Sect. 6.

\section{The sample}

RL09 and RL12 report the detection of extended emission in Spitzer IRAC images of four post-AGB source candidates, namely IRAS 15534-5422, IRAS 17009-4154, IRAS 18229-1127, and IRAS 18454+0001. According to the spectral energy distribution (SED) classification scheme of postAGB sources introduced by van der Veen et al. (1989), the SEDs of these four sources shown in Fig. 1 can be assigned to Type II for IRAS $15534-5422$ (peak at $\sim 25 \mu \mathrm{m}$ and gradual fall-off to shorter wavelengths, although we note that its SED also suggests a near-IR excess at 2-5 $\mu \mathrm{m}$ ), to Type III for IRAS 18229-1127 and IRAS $18454+0001$ (peak at $\sim 25 \mu \mathrm{m}$ and steep fall-off to shorter wavelengths with a plateau between 1 and $4 \mu \mathrm{m}$ ), and to Type IV for IRAS 17009-4154 (with a main peak at $25 \mu \mathrm{m}$ and a secondary peak blue-wards). These SEDs are suggestive of two dust components, cold dust in the thermal IR and hot dust obscuring the central star in the near-IR.

Besides the information provided by RL09 and RL12, there is no detailed study available in the literature for these sources. Based on their IRAS colors, IRAS 15534-5422 is classified as a PN candidate (Preite-Martinez 1988), as recently confirmed by Parker et al. (2012) by means of spectroscopy, whereas IRAS $18454+0001$ is classified as a post-AGB star (García-Lario et al. 1997). We note that the IRAS selection criteria for these sources (Suárez et al. 2006) may overlap with those of young stellar objects (YSOs), although RL09 note that these four objects are not located near star forming regions and they have also not been classified as YSOs in the literature. The lack of CO line emission in IRAS 15534-5422 and 
Table 1. Observation log.

\begin{tabular}{|c|c|c|c|c|c|c|c|c|c|c|}
\hline \multirow[t]{2}{*}{ Object } & \multirow{2}{*}{\multicolumn{2}{|c|}{ 2000.0) }} & \multirow{2}{*}{$\begin{array}{c}\text { Date of } \\
\text { observation }\end{array}$} & \multicolumn{2}{|c|}{ Integration time } & \multirow[t]{2}{*}{ Standard star } & \multicolumn{4}{|c|}{$F W H M$} \\
\hline & & & & $N$ band & $Q$ band & & PAH1 & $\mathrm{SiC}$ & {$[\mathrm{Ne} \mathrm{II}]$} & $\mathrm{Q} 1$ \\
\hline IRAS $15534-5422$ & $15^{\mathrm{h}} 57^{\mathrm{m}} 21^{\mathrm{s}} .11$ & $-54^{\circ} 30^{\prime} 46^{\prime \prime} 4$ & 2011-05-25 & 600 & 900 & HD 133550 & 0.28 & 0.37 & 0.35 & 0.46 \\
\hline IRAS 17009-4154 & $17^{\mathrm{h}} 04^{\mathrm{m}} 29^{\mathrm{s}} .6$ & $-41^{\circ} 58^{\prime} 38^{\prime \prime} .9$ & $2010-05-24$ & 300 & 450 & HD 163376 & 0.31 & 0.36 & 0.38 & 0.48 \\
\hline IRAS $18229-1127$ & $18^{\mathrm{h}} 25^{\mathrm{m}} 45^{\mathrm{s}} .0$ & $-11^{\circ} 25^{\prime} 56^{\prime \prime}$ & 2010-06-14 & 600 & 600 & HD 169916 & 0.34 & 0.51 & 0.42 & 0.49 \\
\hline IRAS $18454+0001$ & $18^{\mathrm{h}} 48^{\mathrm{m}} 01^{\mathrm{s}} .5$ & $+00^{\circ} 04^{\prime} 47^{\prime \prime}$ & $2010-06-22$ & 840 & 1320 & HD 168723 & 0.28 & 0.35 & 0.35 & 0.49 \\
\hline
\end{tabular}

Table 2. Estimated fluxes.

\begin{tabular}{lcccc}
\hline \hline Object & $\begin{array}{c}\text { PAH1 }(8.54 \mu \mathrm{m}) \\
(\mathrm{mJy})\end{array}$ & $\begin{array}{c}\mathrm{SiC}(11.9 \mu \mathrm{m}) \\
(\mathrm{mJy})\end{array}$ & $\begin{array}{c}{[\mathrm{Ne} \mathrm{II}](12.8 \mu \mathrm{m})} \\
(\mathrm{mJy})\end{array}$ & $\begin{array}{c}\text { Q1 }(17.7 \mu \mathrm{m}) \\
(\mathrm{mJy})\end{array}$ \\
\hline IRAS 15534-5422 & $181.6 \pm 0.1$ & $768.5 \pm 0.1$ & $1461.9 \pm 0.1$ & $1891.9 \pm 0.2$ \\
IRAS 17009-4154 & $9054.5 \pm 0.5$ & $4212.2 \pm 0.1$ & $3280.2 \pm 0.2$ & $50674 \pm 5$ \\
IRAS 18229-1127 & $659.78 \pm 0.02$ & $270.25 \pm 0.04$ & $270.79 \pm 0.03$ & $4101.8 \pm 0.1$ \\
IRAS 18454+0001 & $184.34 \pm 0.03$ & $167.61 \pm 0.02$ & $225.17 \pm 0.03$ & $3096 \pm 1$ \\
\hline
\end{tabular}

IRAS $17009-4154$ and the detection of narrow $\left(\simeq 0.8 \mathrm{~km} \mathrm{~s}^{-1}\right)$ CO line emission toward IRAS 18454+0001 (Urquhart et al. 2008, RMS Survey) are inconsistent with a YSO nature. Based on the IRAS colors of the sample, we can also discard a possible symbiotic star nature, as these have typically values of the IRAS [12]-[15] color $\sim 0.8$ (Kenyon et al. 1988), whereas the sources in our sample present values $\geq 2$. Moreover, our sources do not present variability in near-IR observations (RL09 and RL12), so we can disregard an eruptive nature, as for instance in circumstellar shells around luminous blue variable sources (e.g., IRAS 18576+0341, Buemi et al. 2010).

The detection of radio continuum emission in IRAS 15534-5422, IRAS 17009-4154, and IRAS 18454+0001 (Urquhart et al. 2008) confirms that ionization is already present, implying that these three sources may have already entered the PN phase. Indeed, $\mathrm{Br} \gamma$ emission from ionized material has been detected in IRAS 15534-5422 (RL12) and in IRAS 17009-4154 (Van de Steene et al. 2000). For IRAS 15534-5422, this emission is found to be extended and to display a bipolar morphology (RL12). We are thus confident of the PN nature of IRAS 15534-5422, IRAS 17009-4154, and IRAS 18454+0001.

As for IRAS 18229-1127, the absence of filamentary diffuse emission in the mid-IR, which is characteristic of YSOs, and the similarities between its SED and that of IRAS 18454+0001 can be used as additional arguments for a post-AGB classification.

\section{Observations and data reduction}

High angular resolution observations (Program ID: 087.D0367(A), PI: M.A. Guerrero) were obtained with the mid-IR imager VISIR (Lagage et al. 2004) attached to the Cassegrain focus of Melipal (UT3) at the VLT. The sources have been observed through four different filters, PAH1 $\left(\lambda_{\mathrm{c}}=8.54 \mu \mathrm{m}\right.$, $\Delta \lambda=0.42 \mu \mathrm{m}), \operatorname{SiC}\left(\lambda_{\mathrm{c}}=11.85 \mu \mathrm{m}, \Delta \lambda=2.34 \mu \mathrm{m}\right)$ and [Ne II] $\left(\lambda_{\mathrm{c}}=12.82 \mu \mathrm{m}, \Delta \lambda=0.21 \mu \mathrm{m}\right)$ in the $N$ band, and the Q1 filter $\left(\lambda_{\mathrm{c}}=17.65 \mu \mathrm{m}, \Delta \lambda=0.83 \mu \mathrm{m}\right)$ in the $Q$ band. The observations of standard stars were performed after every science ObsBlock to correct for PSF artifacts and flux-calibration purposes. The date of observation, the integration time, and the FWHM of the standard star observed subsequently are summarized in Table 1. The pixel scale is 0 ' $^{\prime} 075$ and the field of view (FoV) is $19 ! 2 \times 19 ! 2$.

The data were taken using several exposures with short detector integration time (DIT) depending on the flux of the source and the required signal-to-noise ratio $(\mathrm{S} / \mathrm{N})$. The observation mode used was the so-called NORMAL with a perpendicular chop-throw of $8^{\prime \prime}$. The chopping and nodding standard technique was used to help in the removal of the background signal. In the NORMAL mode used for these observations, all frames taken at a chopping position are added immediately at the end of the exposure, resulting in a data cube of reduced size. The data reduction was carried out following standard procedures of GasganoVISIR pipeline (version 3.4.4), in which flat-fielding correction, bad pixel removal, source alignment, and co-addition of frames are executed to produce a combined image for each filter. The resulting FoV is $\leq 8^{\prime \prime}$.

To improve the spatial resolution of the raw images, we deconvolved each of them using its PSF observation according to two different deconvolution algorithms: maximum likelihood (number of iterations $\geq 5$ ) and Richardson-Lucy (number of iterations $\geq 10$ ). The precise algorithm and number of iterations was determined by the quality of the raw image, so that the spatial resolution of the deconvolved image was improved, but no artifacts were introduced. The maximum likelihood algorithm was used to deconvolve the images of IRAS 170094154, IRAS 18229-1127, and IRAS 18454+0001, whereas the Richardson-Lucy algorithm was used for IRAS 15534-5422. The final images are presented in Fig. 2.

The final images have been flux-calibrated using fluxes of the PSF stars (Cohen et al. 1999). For the flux calibration we performed aperture photometry (Table 2). We note that the angular extent of IRAS 15534-5422 and IRAS 17009-4154 is similar to the final FoV of $8^{\prime \prime}$. Because the background aperture may include emission from these objects, their fluxes listed in Table 2 should be regarded as lower limits of the real fluxes.

\section{Results}

The VISIR-VLT images presented in Fig. 2 reveal the extended emission from these four sources in unprecedented detail. These images also disclose notable variations among the morphological features shown in images obtained through different filters, which we interpret as related to the spectral features registered by these filters. The emission in the PAH1 filter may include the PAH1 feature at $8.6 \mu \mathrm{m}$ associated with C-rich dust, as well as thermal continuum emission. The broad $\mathrm{SiC}$ filter includes thermal dust emission, silicate features, the PAH2 feature at $11.3 \mu \mathrm{m}$, and emission lines such as [Ne II] and [S IV]. The [Ne II] filter is fine-tuned to the [Ne II] emission from ionized gas, but 

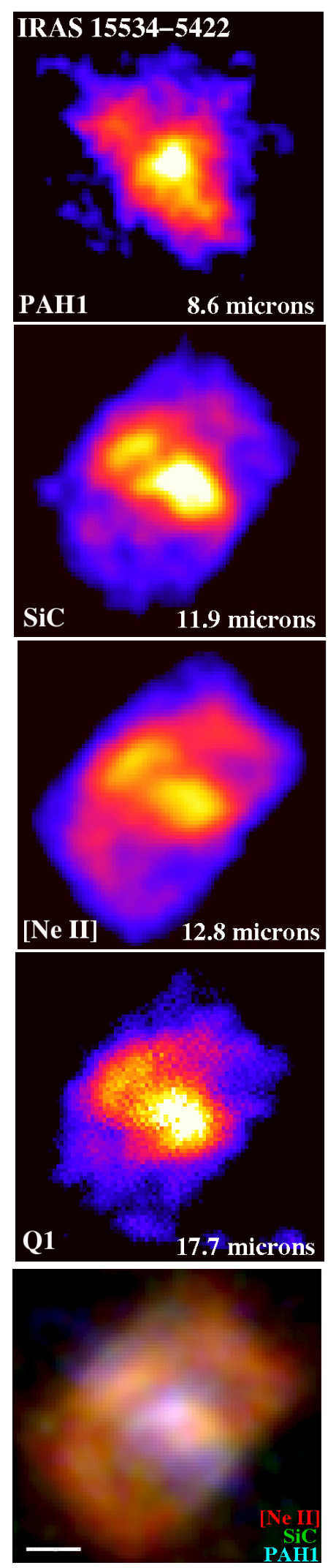
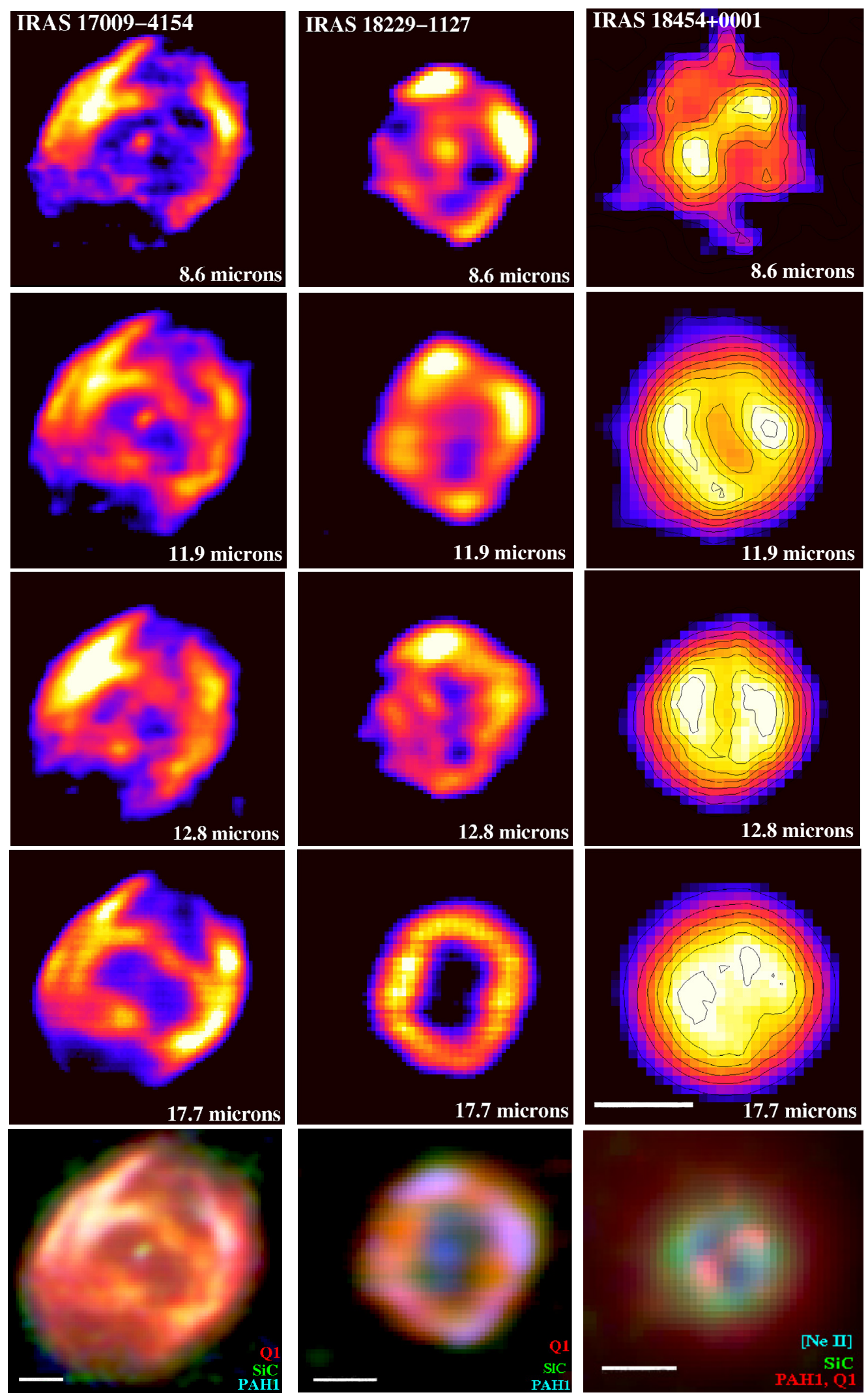

Fig. 2. Deconvolved VISIR images of the objects in our sample in the different filters observed in the $N$ and $Q$ bands. North is up and east to the left. The images of IRAS $18454+0001$ are overlaid with contours at 30, 40,50, 60, 70, 80, 90 and 100\% of the peak intensity to highlight its morphological features. The frames at the bottom are color-composite RGB pictures using the filters with the colors of the corresponding labels. The bar at the bottom of each RGB image and at the bottom of the Q1 image of IRAS 18454+0001 represents 1". The scale of the color-composite picture of IRAS $18454+0001$ is different from that of the individual images to show in the former the faint halo detected in the $Q 1$ band. 

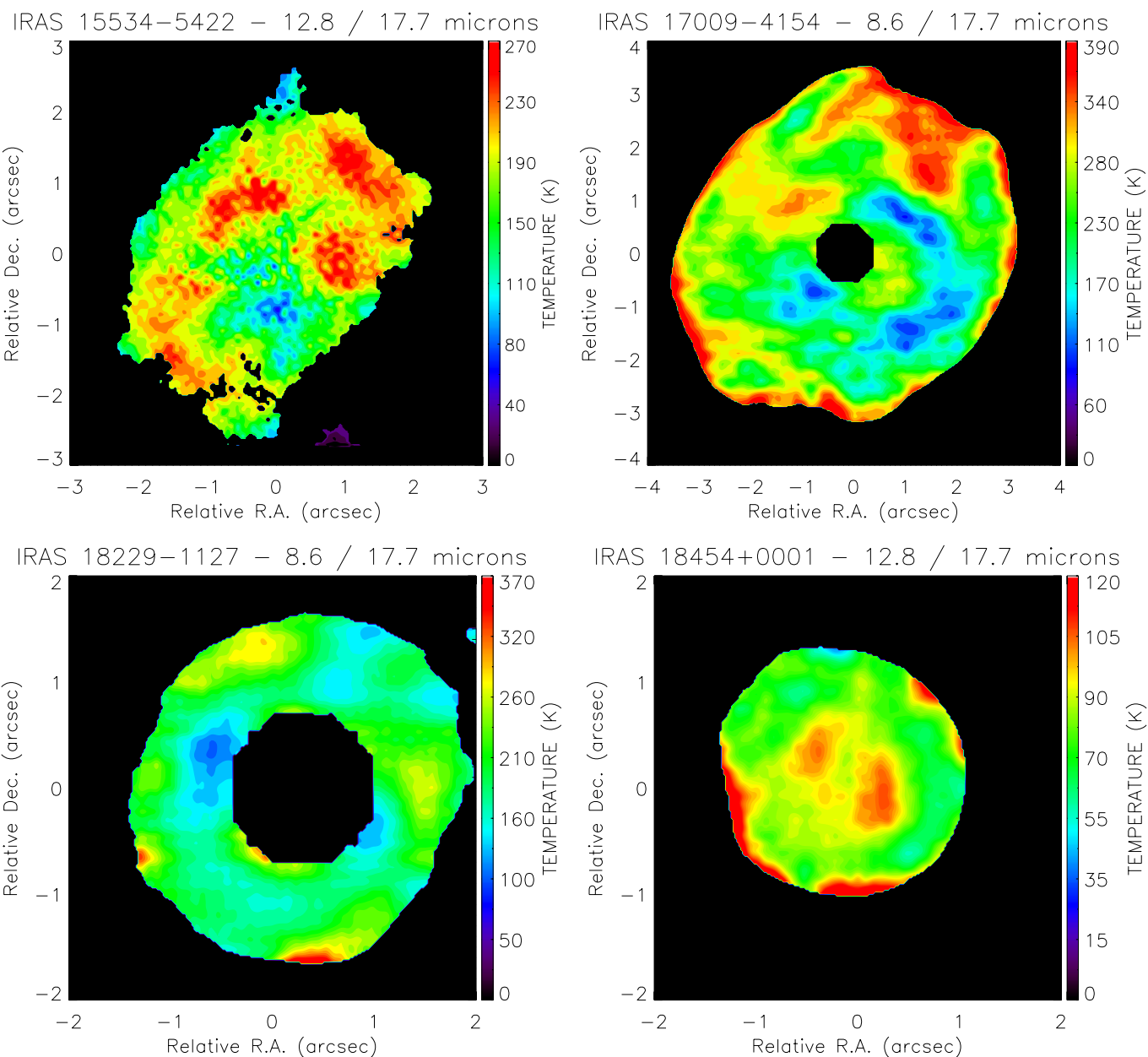

Fig. 3. Temperature (color) maps derived using the Eq. (1) of IRAS 15534-5422, IRAS 17009-4154, IRAS 18229-1127 and IRAS $18454-18454$. We note that the red patches at the borders of the color the maps of IRAS 17009-4154 and IRAS 18454+0001 are artifacts caused by the reduced $\mathrm{S} / \mathrm{N}$ in the outermost regions of these sources. The central stars of IRAS 17009-4154 and IRAS 18229-1127 have been masked to construct the color maps.

it may also include the contribution from dust emission. Finally, the Q1 filter maps the continuum emission of the thermal dust at $17.7 \mu \mathrm{m}$.

Considering that the thermal emission of the dust represents the major contribution to the emission in the mid-IR, we have applied the procedure described by Dayal et al. (1998) and have generated color maps (also known as temperature maps) using pairs of flux calibrated images in different wavelengths for the four objects in our sample (Fig. 3). This procedure relies on the relation between the intensity of the thermal dust continuum at a given wavelength $\left(I_{\lambda}\right)$ with the temperature and optical depth under the assumption of the optically thin emission of the warm dust component. Because the extinction produced by cool dust component along the line of sight can be considered as similar at the mid-IR wavelengths of the different filters used in this work, we can further assume that its effects on the ratio maps are negligible. Since variations in the temperature produced by the emission properties of the dust appear as color variations in the maps, the temperature of the dust can be approximated by the following expression:

$T \approx \frac{1.44 \times 10^{4}\left(1 / \lambda_{2}-1 / \lambda_{1}\right)}{\ln \left[\left(I_{\lambda_{1}} / I_{\lambda_{2}}\right)\left(\lambda_{1} / \lambda_{2}\right)^{3}\right]} \mathrm{K}$

where $\lambda_{1}$ and $\lambda_{2}$ are the two wavelengths used for the estimation of the temperature, and $I_{\lambda_{1}}$ and $I_{\lambda_{2}}$ are the measured intensities at these two wavelengths. This method assumes that the variation in the emissivity $(Q)$ can be represented by a power law $\left(Q \sim \lambda^{-n}\right)$, and it has been proven to provide a good estimate of the spatial distribution of the cold and warm dust across the source studied (Dayal et al. 1998; Meixner et al. 1999; Ueta et al. 2001; Lagadec et al. 2005), since it is helpful to reveal or enhance morphological features.

To derive the color maps shown in Fig. 3, we used the Q1 image in conjunction with one $\mathrm{N}$-band image, since the longer wavelength range provides a better leverage of the continuum slope that is determined by the thermal dust temperature. We also avoided images in those filters that may present important contributions of spectral features other than the continuum. Therefore, the $\mathrm{SiC}$ images were not used, because they may include multiple contributions from emission lines and dust features.

Once the value of the temperature is estimated, we can construct optical depth maps using the Planck function under the same assumption. Therefore, the optical depth at a certain wavelength $\lambda$ can be derived using the following expression:

$\tau_{\lambda} \approx-\ln \left[1-\frac{I_{\lambda}}{B_{\lambda}(T)}\right]$

where $I_{\lambda}$ is the intensity at the wavelength analyzed. Using these maps, we are able to estimate the column density variations across the nebula to assess whether the emission is optically thin at the observed wavelength range. 

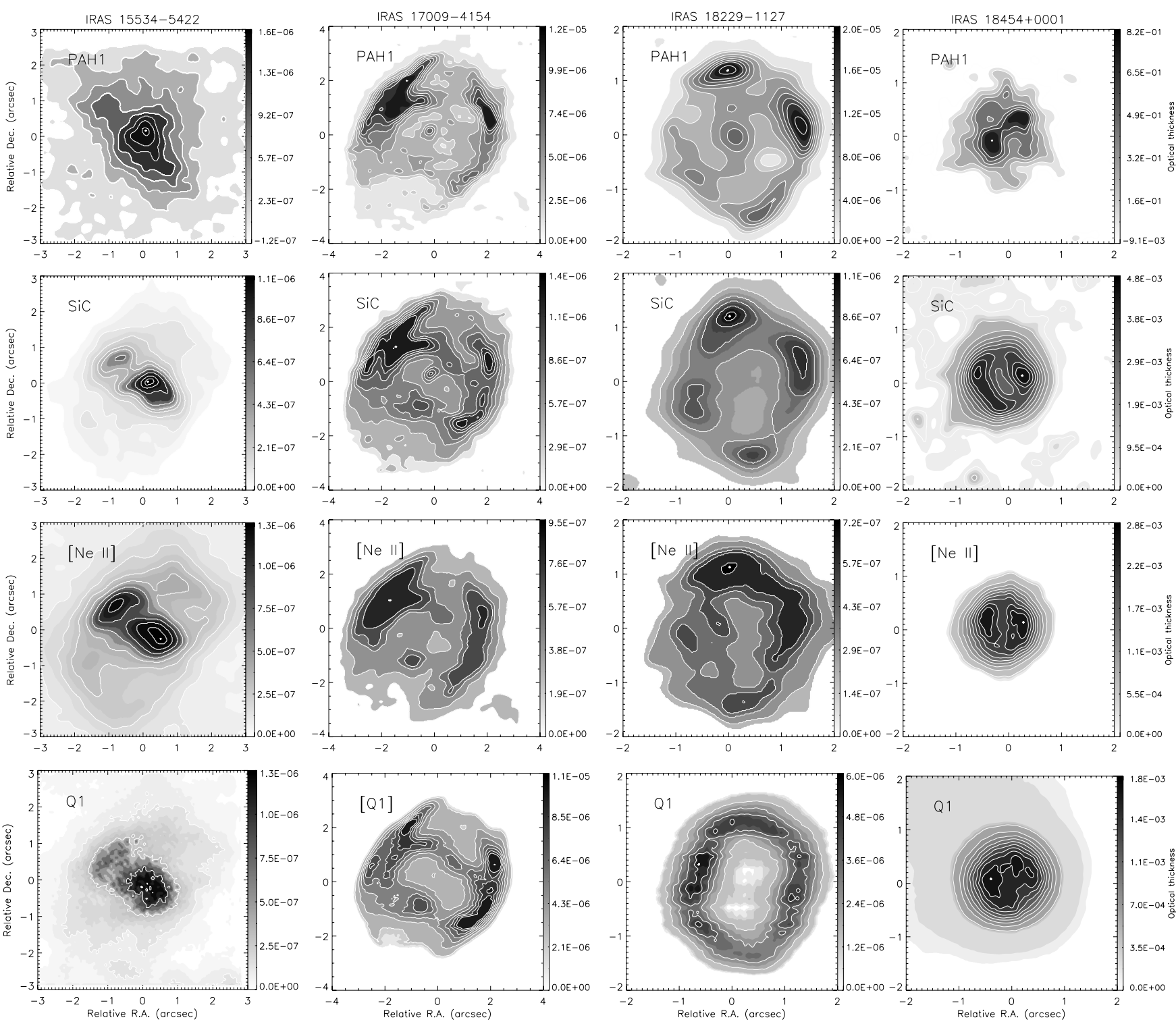

Fig. 4. Optical depth maps of the sources obtained using Eq. (2) for the four different wavelengths observed. Contours are in intervals of $10 \%$ of the intensity peak for IRAS 17009-4154, IRAS 18229-1127, and IRAS 18454+0001. For IRAS 15534-5422 the contours are in intervals at 10\% for [Ne II] and SiC, intervals at $20 \%$ for Q1, and for the PAH1 map the contours start at $70 \%$ of the intensity in intervals of $2.5 \%$ of the intensity peak.

We next describe in detail the morphology, temperature, and optical depth properties of the individual sources.

\subsection{IRAS 15534-5422}

IRAS $15534-5422$ is resolved in at least two structures that are distinguished by their morphology and emission properties (Fig. 2). A first structure is an elongated, bar-like feature with a size of $\sim 3^{\prime \prime}$ oriented at PA $55^{\circ}$. The emission of this bar is detected in PAH, SiC, and Q1, and is much weaker in [Ne II]. A second structure is an arc-like feature, $\sim 5^{\prime \prime} \times 3^{\prime \prime}$ in size, which is oriented perpendicular to the bar and is particularly prominent in [Ne II], but weak in SiC and Q1 and absent in PAH1. This arc-like extended structure is not closed and presents a rectangular shape at low intensity levels. The color composite picture of IRAS 15534-5422 (Fig. 2) suggests that the arc-like feature traces ionized material, while the bar is mostly dominated by thermal dust emission.
The color map of this source (Fig. 3), obtained using the [Ne II] and the Q1 images, reveals a range of temperatures across the nebula from $80 \pm 1 \mathrm{~K}$ to $270 \pm 1 \mathrm{~K}$, with a mean value of $\approx 190 \mathrm{~K}$. The color map greatly enhances the arc-like feature, which is the hottest component, whereas the bar, with lower temperatures, almost disappears in this map. The optical depth maps at different wavelengths (Fig. 4), computed for a mean temperature value of $190 \mathrm{~K}$, imply that the south-west tip of the bar represents the highest density zone in this object. The [Ne II] optical depth map reveals a noticeable enhancement of the column density at the northeast tip of the bar, where it goes across the arc-like feature.

\subsection{IRAS $17009-4154$}

IRAS 17009-4154 has an elongated morphology toward the northwest with a size of $6.3 \times 55^{\prime \prime} 5$. There is an innermost equatorial enhancement that can be appreciated as an elongated 
ring-like structure $\left(\sim 3^{\prime \prime} \times 2^{\prime \prime}\right)$ at $\mathrm{PA} \sim 64^{\circ}$ surrounding a central star that is detected in all VISIR bands (Fig. 2). Two prominent arc-like features trace the edge of the outermost regions and are located at PAs $64^{\circ}-244^{\circ}$ with brightness increasing with wavelength.

The color map of this source (Fig. 3) shows values of temperature in the range from $150 \pm 5 \mathrm{~K}$ up to $390 \pm 5 \mathrm{~K}$ with a mean value of $210 \mathrm{~K}$. The maxima in temperature are related to regions at the tips of the major axis of symmetry. The innermost ringlike structure surrounding the central star seems to have two different temperature components: the southwest is the coolest region $(\sim 150 \mathrm{~K})$, whereas there is a hot component located toward northeast with a temperature of $\sim 350 \mathrm{~K}$. The arcs are warmer $(\sim 250 \mathrm{~K})$. The mean value of the temperature $(T=210 \mathrm{~K})$ has been used to derive the optical depth maps of IRAS 17009-4154 (Fig. 4). The regions with the highest column densities are the arcs, followed by the ring-like structure, as shown in all optical depth maps.

\subsection{IRAS 18229-1127}

IRAS 18229-1127 has a rhomboidal clumpy envelope with an angular size of 2 .' 5 . In the PAH1, SiC, and [Ne II] $N$-band images (Fig. 2), this envelope is dominated by two bright knots toward the north and northwest, and two weaker knots are noticeable toward the south and southeast. The southeast knot is not detected in [Ne II]. Interestingly, these knots are singularly not apparent in the Q1 image that shows a clearly hollow envelope, with an elongated cavity oriented along the north-south direction. The central star is detected, but only in the bluest PAH1 image at $8.6 \mu \mathrm{m}$. The color-composite RGB picture of this object at the bottom of Fig. 2 shows a reddened, hollow rhomboidal envelope with a spot brighter at shorter wavelengths at each corner.

We derived the color map of this source using its PAH1 and Q1 images and estimated a mean temperature of $170 \mathrm{~K}$ (Fig. 3), with a range of $100 \mathrm{~K} \leq T \leq 370 \pm 1 \mathrm{~K}$.

The bright knots in the $N$-band filters are the warmest regions of this source, with $T \simeq 280 \mathrm{~K}$, whereas the rest of the rhomboidal envelope has the lowest temperatures $(\sim 200 \mathrm{~K})$. The mean value of $170 \mathrm{~K}$ has been used to derive the optical depth maps of IRAS 18229-1127 (Fig. 4). The knots represent the regions with the highest densities in the $N$-band, with the north one the densest. These density variations are diminished in the Q1 optical depth map, where the rhomboidal envelope has a more homogeneous density distribution. The difference between the optical depth maps of the $N$-band and the one of Q1 is remarkable: while the knots are the densest zones from 8.6 to $12.8 \mu \mathrm{m}$, the peak of the density at $17.7 \mu \mathrm{m}$ is toward the east, where not a single knot is present.

\subsection{IRAS 18454+0001}

This source has the smallest angular size among the objects in our sample. The source can be described as a round clumply envelope of 1".5 in size, clumpy envelope in the $\mathrm{SiC},[\mathrm{Ne} I \mathrm{II}$, and Q1 images, but its morphology in the PAH1 image is singularly different (Fig. 2). In this band, the image is dominated by a pair of bright knots along PA $121^{\circ}$ that seem spatially coincident with brightness peaks in the Q1 image, whereas the peaks detected in the $\mathrm{SiC}$ and $[\mathrm{Ne} \mathrm{II}]$ images are located at different positions. All images hint at the presence of a central cavity, but its orientation and extent varies with wavelength. On a large spatial scale, the Q1 image reveals an extended halo around the main nebula.
The halo with a size of $5^{\prime \prime} \times 4^{\prime \prime}$ extends toward the northeast, as shown in red in the color-composite RGB picture at the bottom of Fig. 2.

The [Ne II] and Q1 images of IRAS 18454+0001 were used to estimate the mean value of temperature of $80 \mathrm{~K}$ in a range from $50 \pm 1 \mathrm{~K}$ to $120 \pm 1 \mathrm{~K}$ (Fig. 3). The temperature variations are notably flat over the nebula, with the hottest temperature $(\gtrsim 100 \mathrm{~K})$ at the pair of bright spots seen in [Ne II] and regions of high temperature $(\simeq 90 \mathrm{~K})$ at the bright PAH1 and Q1 knots. The emission of the envelope seems to be traced by a cooler dust component. Interestingly, the optical depth maps (Fig. 4) show that the highest density regions are associated with the knots seen in PAH1 and in Q1, while the knots associated with the $[\mathrm{Ne}$ II] emission peaks represent zones of lower column density. There is a remarkable difference in the value and location of the peaks in the the optical depth maps of IRAS 18454+0001 in PAH1 and Q1 with respect to those in $\mathrm{SiC}$ and [Ne II], but we note this may be an artifact produced by the low value of the temperature estimated for this source $(T=80 \mathrm{~K})$ that has been used to built its optical depth maps.

\section{Discussion}

\subsection{Physical structure of the sources}

We obtained mid-IR high spatial resolution images of three PNe and one post-AGB source candidate using VISIR at the VLT. These images reveal notable morphological differences among the sources in our sample. The shell-like morphology and detection of a central star in three of these sources are not expected for the diffuse mid-IR emission from compact $\mathrm{H}$ II regions (Takahashi et al. 2000). Only IRAS 15534-5422 may look like a compact $\mathrm{H}$ II region, but its PN nature is well established by optical spectroscopy (Parker et al. 2012).

Temperature and optical depth maps built from these images have been proven to be powerful tools to enhance morphological structures and to reveal new ones in these objects. In the following we discuss the main characteristics of the individual sources, as well as the characteristics that are shared by them all.

IRAS $15534-5422$ is a young PN with two distinct structural components. The elongated innermost structure is dominated by thermal dust continuum, and it represents the coldest and densest regions in this PN. This structure is surrounded by a warmer, ionized extended envelope of lower density. Whereas the colorcomposite picture of IRAS 15534-5422 in Fig. 2 seems to imply that the elongated inner structure is a bipolar nebula surrounded by the ring-like envelope, the physical properties of the different components favor an alternative, more solid interpretation. The innermost structure is instead a high-density, dusty "torus", whereas the apparent envelope can be associated to a pair of ionized bipolar lobes perpendicular to that "torus". This interpretation is consistent with the narrow-band near-IR images presented by Ramos-Larios et al. (2012) and with the near-IR excess noticeable in its SED (Fig. 1).

IRAS 17009-4154 seems to have an slightly bipolar or elliptical morphology. The bright arcs can be interpreted as ionized bipolar extensions of an elliptical shell sculpted by an $\mathrm{SiC}$ and Q1 bright, dusty equatorial ring. The dust ring is the coldest structure in this source, whereas the bright arcs are warmer. The temperature is the highest at the northwest and southeast tips of the lobes.

Based on the properties of the SED of IRAS 18229-1127 and on its similarity with that of the PN IRAS 18454+0001 (Fig. 1), we favor its classification as a post-AGB source and 
discard a YSO nature. This source shows a peculiar clumpy and dusty rhomboidal envelope. This rhomboidal appearance is enhanced by the knots located toward the northwest, the densest region of this source, and southeast knots. The rhomboidal morphology of IRAS 18229-1127 resembles the shape of the PN BD+303639 (Lagadec et al. 2011; Akras \& Steffen 2012).

IRAS $18454+0001$ is a compact PN displaying both a spherical AGB shell and a dusty torus, which are most noticeable in the PAH1 $(8.6 \mu \mathrm{m})$ and Q1 $(17.7 \mu \mathrm{m})$ images. The temperature map suggests the presence of relatively hot regions along the direction perpendicular to this torus. Alternatively, these regions may be dominated by ionized gas unveiled by its [Ne II] emission. The torus shows density enhancements in the dust-dominated PAH1 and Q1 filters corresponding with the high density knots along the southeast and northwest direction. The presence of a high density torus, but a spherical AGB envelope, is intriguing. We might witness in this source the early phase on the shaping of a bipolar PN, before the spherical geometry of the AGB wind is disrupted by a fast stellar wind collimated by a toroidal density enhancement as proposed by the generalized interacting stellar wind model of PN shaping (GISW, Balick et al. 1987).

The morphological features detected in direct images are notably enhanced in the temperature and optical depth maps, because these distinguish between structures of the same brightness, but different physical conditions. Furthermore, the physical characteristics of the structures detected for each source are generally consistent. The innermost dusty rings or torii of IRAS 15534-5422, IRAS 17009-4154, and IRAS 18454+0001 have lower temperatures $(T \sim 100 \mathrm{~K})$ than the elongated hotter $(T \gtrsim 150 \mathrm{~K})$ bipolar regions.

The optical depth maps of IRAS 15534-5422, IRAS 170094154, and IRAS 18229-1127 peak at similar values for each of these sources. This implies that there are no variations in the density derived from the different filters for each of these sources, and thus that the mid-IR emission of these source is optically thin.

\subsection{Comparison with the morphology and physical structure of other evolved objects}

Our mid-IR VISIR-VLT images have unveiled a wealth of structural components in the four sources in our sample. In short, these sources can be described as a detached rhomboidal shell (IRAS 18229-1127), a spherical shell with strong evidence of a dusty torus orthogonal to an ionized bipolar flow. (IRAS 18454+0001), and two mild bipolar or elliptical sources (IRAS 15534-5422 and IRAS 17009-4154). Unlike other studies of sources in the transition towards the PN phase (Sahai \& Trauger 1998; Ueta et al. 2000; Sahai et al. 2007, 2011; Lagadec et al. 2011), we do not find evidence of extreme axisymmetric bipolar or multipolar morphologies. In this respect, we note that we have not imposed on the sources in our sample any of the restrictions of previous studies, required the sources to be detected in the optical (Ueta et al. 2000; Sahai et al. 2011), or to have fluxes at $12 \mu \mathrm{m}$ greater than $10 \mathrm{Jy}$ (Lagadec et al. 2011). These selection criteria may have introduced notorious biases in the morphological output of the sample.

In this sense, the lack of restrictive selection criteria in our sample may probe a different population of sources in the transition between the late AGB and early post-AGB phases. The small number of objects in our sample does not allow us to drawn firm conclusions; however, the observed morphologies may be analyzed within the evolutionary context of PN formation. At least three of the sources in our sample (IRAS 15534-5422, IRAS 17009-4154, and IRAS 18454+0001) have already reached the PN stage, although they are still mostly obscured at optical wavelengths. The significant obscuration can be attributed to large amounts of circumstellar material that has been previously ejected by their central stars, suggesting massive progenitors. Interestingly, the optical depth values computed for IRAS $18454+0001$ are significantly higher than those of the remaining sources, whereas its temperature is lower. The thicker envelope of this source may be indicative of an even more massive progenitor.

If the sources in our sample indeed descended from massive AGB stars, we do not see the expected correlation between extreme bipolar morphology and massive progenitors (Corradi \& Schwarz 1995; Ueta et al. 2000; Siódmiak et al. 2008). It can be argued that the sources in our sample are at a very early evolutionary phase, when asymmetries have not fully developed yet. For example, IRAS 15534-5422, with its dense, dusty torus and bipolar lobes, might be a bipolar PN in the making, whereas the high density torus of IRAS $18454+0001$, enclosed within a spherical shell, can provide the seed for a bipolar PN.

The use of color maps in the mid-IR domain has revealed a torus in IRAS 07134+1005 (Dayal et al. 1998) and hinted at the presence of jets in Roberts 22 and in V Hya (Lagadec et al. 2005). The mid-IR size of these sources $\left(\sim 4^{\prime \prime}\right)$ are similar to that of the sources in our sample, but the variations in the temperature across the sources studied in this work have shown structures that define the morphology more clearly. Compared with these previous analyses of color and optical depth maps, our study resolves many more morphological details, especially if we note that the sources in our sample do not display extreme axisymmetric nebulosities. The high-spatial resolution achieved by our data is certainly required to resolve the extended emission of the obscured and small-sized or compact sources transiting this evolutionary phase.

\section{Conclusions}

We have observed four heavily obscured post-AGB sources and PN candidates with VISIR-VLT in three different $N$ bands (PAH1, SiC, and [Ne II]), and one $Q$ band (Q1). Three sources in our sample (IRAS 15534-5422, IRAS 17009-4154, and IRAS 18454+0001) can be classified as young PNe, whereas only a preliminary classification as a post-AGB source is possible for IRAS 18229-1127.

The high-spatial resolution VISIR images were used to investigate the extended emission and to study the spatial variations of the physical conditions (temperature and optical depth) of these sources. We provided evidence of asymmetry in three young PNe: an innermost dust torus or ring embedded within an ionized spherical shell (IRAS 18454+0001), and two mild bipolar or elliptical sources with dusty rings (IRAS 15534-5422 and IRAS 17009-4154).

Compared to previous works, the use of color and optical depth maps have proven very useful in revealing fine structural details in a small sample of heavily obscured sources reaching the PN phase, confirming the usefulness of mid-IR highresolution observations for the study of this short evolutionary phase.

Our imaging study confirmed that asymmetry is present in heavily obscured post-AGB stars and young PNe but, unlike previous studies focused on these evolutionary phases, no extreme axisymmetric morphologies are found. These previous studies may be biased towards the mid-IR and optical brightest sources, missing critical early phases of the post-AGB evolution of the 
most massive progenitors. Although based on a small sample, our study may be yielding important clues to the onset of asymmetry in massive progenitors of PNe. Further studies of the most obscured post-AGB sources must be pursued in the future.

Acknowledgements. Part of this work was supported by the Ministerio de Economía y Competitividad of Spain through grants AYA 2008-01934, AYA 2008-06189-C03-01, AYA 2011-29754-C03-02, and AYA 2011-30228CO3-01 cofunded by FEDER funds. MWB would like to thank the EEBB-FPI for the grant for the short term stay in 2011, and thank the European Southern Observatory (ESO) Headquarters in Garching, Germany, for all the facilities provided during this stay. G.R.L. acknowledges support from CONACyT (grant 177864) and PROMEP (Mexico). L.F.M. is also supported by grant IN845B2010/061 of Xunta de Galicia, partially funded by FEDER funds. This paper made use of information from the red MSX Source survey database at http: // www.ast.leeds.ac.uk/RMS, which was constructed with support from the Science and Technology Facilities Council of the UK. We would like to thank the referee, Dr. C. Waelkens, for his useful comments for improving of this paper.

\section{References}

Akras, S., \& Steffen, W. 2012, MNRAS, 423, 925

Balick, B., \& Frank, A. 2002, ARA\&A, 40, 439

Balick, B., Preston, H. L., \& Icke, V. 1987, AJ, 94, 1641

Buemi, C. S., Umana, G., Trigilio, C., Leto, P., \& Hora, J. L. 2010, ApJ, 721, 1404

Cohen, M., Walker, R. G., \& Witteborn, F. C. 1999, LPI Contributions, 969, 5

Corradi, R. L. M., \& Schwarz, H. E. 1995, A\&A, 293, 871

Cox, N. L. J., García-Hernández, D. A., García-Lario, P., \& Manchado, A. 2011, AJ, 141, 111

David, P., Le Squeren, A. M., \& Sivagnanam, P. 1993, A\&A, 277, 453

Dayal, A., Hoffmann, W. F., Bieging, J. H., et al. 1998, ApJ, 492, 603

Jiménez-Esteban, F. M., García-Lario, P., Engels, D., \& Perea Calderón, J. V. 2006, A\&A, 446, 773

de Gregorio-Monsalvo, I., Gómez, Y., Anglada, G., et al. 2004, ApJ, 601, 921
García-Lario, P., Manchado, A., Pych, W., \& Pottasch, S. R. 1997, A\&AS, 126, 479

Kenyon, S. J., Fernandez-Castro, T., \& Stencel, R. E. 1988, AJ, 95, 1817

Lagadec, E., Mékarnia, D., de Freitas Pacheco, J. A., \& Dougados, C. 2005, A\&A, 433, 553

Lagadec, E., Verhoelst, T., Mékarnia, D., et al. 2011, MNRAS, 417, 32

Lagage, P. O., Pel, J. W., Authier, M., et al. 2004, The Messenger, 117, 12

Manchado, A., Villaver, E., Stanghellini, L., \& Guerrero, M. A. 2000, Asymmetrical Planetary Nebulae II: From Origins to Microstructures, 199, 17

Meixner, M., Ueta, T., Dayal, A., et al. 1999, ApJS, 122, 221

Parker, Q. A., Cohen, M., Stupar, M., et al. 2012, MNRAS, 427, 3016

Preite-Martinez, A. 1988, A\&AS, 76, 317

Ramos-Larios, G., Guerrero, M. A., Suárez, O., Miranda, L. F., \& Gómez, J. F. 2009, A\&A, 501, 1207

Ramos-Larios, G., Guerrero, M. A., Suárez, O., Miranda, L. F., \& Gómez, J. F. 2012, A\&A, 545, A20

Sahai, R., \& Trauger, J. T. 1998, AJ, 116, 1357

Sahai, R., Morris, M., Sánchez Contreras, C., \& Claussen, M. 2007, AJ, 134, 2200

Sahai, R., Morris, M. R., \& Villar, G. G. 2011, AJ, 141, 134

Schwarz, H. E., Corradi, R. L. M., \& Stanghellini, L. 1993, Planetary Nebulae, 155,214

Siódmiak, N., Meixner, M., Ueta, T., et al. 2008, ApJ, 677, 382

Suárez, O., García-Lario, P., Manchado, A., et al. 2006, A\&A, 458, 173

Suárez, O., Lagadec, E., Bendjoya, P., et al. 2011, Asymmetric Planetary Nebulae 5 conference, held in Bowness-on-Windermere, U.K., 20-25 June 2010, eds. A. A. Zijlstra, F. Lykou, I. McDonald, \& E. Lagadec, Jodrell Bank Centre for Astrophysics

Takahashi, H., Matsuhara, H., Watarai, H., \& Matsumoto, T. 2000, ApJ, 541, 779

Ueta, T., Meixner, M., \& Bobrowsky, M. 2000, ApJ, 528, 861

Ueta, T., Meixner, M., Dayal, A., et al. 2001, ApJ, 548, 1020

Urquhart, J. S., Hoare, M. G., Lumsden, S. L., Oudmaijer, R. D., \& Moore, T. J. T. 2008, Massive Star Formation: Observations Confront Theory, 387, 381

Van de Steene, G. C., van Hoof, P. A. M., \& Wood, P. R. 2000, A\&A, 362, 984 van der Veen, W. E. C. J., Habing, H. J., \& Geballe, T. R. 1989, A\&A, 226, 108 